\title{
Review of: "Directed nickel-catalyzed regio- and diastereoselective arylamination of unactivated alkenes"
}

\author{
Zhuangzhi Shi ${ }^{1}$ \\ 1 Nanjing University
}

Potential competing interests: The author(s) declared that no potential competing interests exist.

The Wang group described a Ni(II)-catalyzed regio- and diastereoselective 1,2-arylamination of unactivated alkenyl amines containing an easily removed PA-directing group. This approach exhibited a remarkably extensive alkenyl amine scope (e.g. allylic amines, homoallylic amines and bishomoallylic amines), primary and secondary amine sources and arylboronic acid scope with excellent functional group tolerance. This arylamination strategy provides a more effective way to simultaneously construct C-C and C-N bonds. The paper also showed good practicality of the reaction: 1) extended to gram-scale, 2) applied to site-selective modification of pharmaceutically derived analogues.

The new methodology with excellent functional group tolerance and high levels of regio-, chemo-, and diastereocontrol are encouraging. Particularly, the reaction could afford rapid access to branched molecules bearing two skipped and vicinal stereocenters with high dr, while previously reported methods could only diastereoselectively furnish products with two vicinal stereocenters.

I find the work impressive and will have a positive impact on the area of alkene functionalization. As such, I think this paper is well fit for Nature Communications with good reputations. 